\title{
Análise da seletividade alimentar de crianças com Transtorno do Espectro Autista
}

\author{
Analysis of the food selectivity of children with Autistic Spectrum Disorder \\ Análisis de la selectividad alimentaria de niños con trastorno del espectro autista
}

\begin{abstract}
Gilma Sannyelle Silva Rocha ${ }^{1}$, Francisco Cesino de Medeiros Júnior ${ }^{1}$, Najra Danny Pereira Lima$^{1}$, Marcus Vinicius da Rocha Santos da Silva ${ }^{2^{*}}$, Andressa da Silva Machado ${ }^{1}$, Irislene Costa Pereira ${ }^{3}$, Mayanny da Silva Lima ${ }^{1}$, Natália Monteiro Pessoa ${ }^{1}$, Sannya Cianna Silva Rocha ${ }^{1}$, Hailany Araújo Costa da Silva ${ }^{1}$.
\end{abstract}

\section{RESUMO}

Objetivo: Analisar a possível presença de comportamentos de seletividade alimentar em crianças com Transtorno do Espectro Autista (TEA). Métodos: Tratou-se de uma pesquisa descritiva, do tipo exploratória, com abordagem quantitativa. O cenário desta investigação foi o Município de Caxias, situado na região leste do estado do Maranhão, utilizou-se como campo de pesquisa a Associação de Pais e Amigos dos Excepcionais (APAE). Foi utilizado como material de coleta de dados um questionário com perguntas fechadas a respeito de aspectos alimentares. Resultados: Os resultados deste estudo apontam que os participantes possuem comportamentos tendenciosos à seletividade alimentar. O principal comportamento identificado na alimentação foi a repetição dos mesmos alimentos consumidos e dificuldades com a textura que eles apresentam. Conclusão: Comportamentos de seletividade alimentar foram identificados na amostra estudada. Sugere-se que estudos posteriores investiguem a presença de seletividade alimentar e consumo de micronutrientes em pessoas com TEA.

Palavras-chave: Transtorno do Espectro do Autismo (TEA), Seletividade Alimentar, Estado Nutricional.

\begin{abstract}
Objective: To analyze the possible presence of food selectivity behaviors in children with Autism Spectrum Disorder (ASD). Methods: This was a descriptive, exploratory type study with a quantitative approach. The setting of this research was the Municipality of Caxias, located in the eastern region of the state of Maranhão. The Association of Parents and Friends of the Exceptional (APAE) was used as a research field. A questionnaire with closed questions about food aspects was used as data collection material. Results: The results of this study indicate that the participants have tendentious behaviors to food selectivity. The main behavior identified in the diet was the repetition of the same foods consumed and difficulties with the texture they present. Conclusion: Food selectivity behaviors were identified in the studied sample. It is suggested that later studies investigate the presence of food selectivity and micronutrient consumption in people with ASD.
\end{abstract}

Key words: Autism Spectrum Disorder (ASD), Food Selectivity, Nutritional Status.

\footnotetext{
${ }^{1}$ Centro Universitário de Ciências e Tecnologia do Maranhão (UniFacema). Caxias-MA.

²Universidade Federal do Maranhão (UFMA). Caxias-MA. *E-mail: marcusvinicius.darocha@yahoo.com.br

${ }^{3}$ Universidade Federal do Piauí (UFPI). Teresina-PI.
}

SUBMETIDO EM: 2/2019

ACEITO EM: 3/2019

PUBLICADO EM: 5/2019

REAS/EJCH | Vol.Sup.24 | e538 | DOI: https://doi.org/10.25248/reas.e538.2019 Página 1 de 8 


\section{RESUMEN}

Objetivo: Analizar la posible presencia de comportamientos de selectividad alimentaria en niños con Trastorno del Espectro Autista (TEA). Métodos: Se trata de una investigación descriptiva, del tipo exploratorio, con abordaje cuantitativo. El escenario de esta investigación fue el Municipio de Caxias, situado en la región este del estado de Maranhão, se utilizó como campo de investigación la Asociación de Padres y Amigos de los Excepcionales (APAE). Se utilizó como material de recolección de datos un cuestionario con preguntas cerradas acerca de aspectos alimentarios. Resultados: Los resultados de este estudio apuntan que los participantes poseen comportamientos tendenciosos a la selectividad alimentaria. El principal comportamiento identificado en la alimentación fue la repetición de los mismos alimentos consumidos y dificultades con la textura que presentan. Conclusión: Comportamientos de selectividad alimentaria fueron identificados en la muestra estudiada. Se sugiere que estudios posteriores investiguen la presencia de selectividad alimentaria y consumo de micronutrientes en personas con TEA.

Palabras clave: Trastorno del Espectro del Autismo (TEA), Selectividad Alimentaria, Estado Nutricional.

\section{INTRODUÇÃO}

O Transtorno do Espectro Autista (TEA) é um transtorno do desenvolvimento que pode ser reconhecido por déficits clinicamente significativos e persistentes na comunicação social e nas interações sociais; déficits expressivos na comunicação não verbal e verbal; padrões restritos e repetitivos de comportamento, interesses e atividades (APA, 2014).

Existe uma habitual discussão na literatura sobre a melhor aferição da prevalência de TEA. A publicação mais recente, de 2014, do conjunto de pesquisas engendradas pelo consagrado Center for Disease Control and Prevention (CDC) mostrou uma prevalência de 14.7 por 1.000 (1 a cada 68) crianças afetadas por TEA nos Estados Unidos (índice 30\% superior a do estudo anterior publicado em $2012 \mathrm{com}$ a mesma metodologia), que ocorrem em todos os grupos raciais, étnicos e socioeconômicos (ELSABBAGH M, et al., 2012; BAXTER AJ, et al., 2015).

Entretanto, as informações americanas do CDC têm retratado valores mais acentuados que as obtidas pela maioria das outras investigações desenvolvidas nos Estados Unidos e em outras localidades do mundo. Em síntese, averiguações nupérrimas indicam uma elevação nas estimativas de números de prevalência de TEA, sendo a melhor estimativa de 0,6\% e 1\% (ELSABBAGH M, 2012; BAXTER AJ, et al., 2015).

Sobre essa temática, salienta-se que, no Brasil, ainda não há dados epidemiológicos significantes, sendo que um estudo piloto concluído e publicado recentemente indica uma prevalência de 1:360 (2,7 por 1000), embora se acredite que tal estimativa esteja subestimada (PAULA CS, et al., 2011).

Dentre as alterações comportamentais presente nos quadros de TEA, a literatura (SILVA NI, 2011; WESTWOOD H, et al., 2017) destaca a seletividade alimentar. A seletividade alimentar pode ser entendida como um comportamento alimentar que tem como característica principal a exclusão de uma variedade de alimentos. Essa postura, muitas vezes, pode ser transitória, (correspondendo à fase de adaptação a novos alimentos), ou perdurar ao longo do desenvolvimento da pessoa (SAMPAIO ABM, et al., 2013).

A Seletividade Alimentar caracteriza-se pela tríade: pouco apetite, recusa alimentar e desinteresse pelo alimento. Essa combinação pode provocar uma certa limitação a variedades de alimentos ingeridos, além disso provoca um comportamento de resistência em experimentar novos alimentos. A limitação de variedades na hora da refeição pode agregar carências nutricionais e prejudicar o organismo, pois a ingestão de macro e micronutrientes está estreitamente relacionada com a ingestão de energia e bom funcionamento do organismo (DOMINGUES G, 2011).

Uma nutrição adequada ajuda na prevenção de doenças, no bom funcionamento do organismo, além de proporcionar uma melhor qualidade de vida. Para a manutenção de uma nutrição adequada é necessário 
que o indivíduo consuma uma variedade de alimentos, pois essa variedade traz uma maior oferta de nutrientes. Crianças com TEA podem apresentar dificuldades em aceitar novas experiências alimentares, 0 que pode ocasionar deficiência de algum nutriente (SILVA NI, 2011).

Os resultados de um amplo estudo, que analisou seletividade alimentar de 138 crianças autistas comparadas a 298 crianças típicas entre 5 a 9 anos, indicaram que as crianças autistas recusavam significativamente mais alimentos e tinham uma dieta menos variada. Concluindo que crianças autistas apresentam mais seletividade alimentar que crianças típicas e que esse comportamento pode ter associação com o estado nutricional (SCHRECK KA, et al., 2004).

Ademais, alguns estudos apontam que a sensibilidade sensorial pode levar as crianças com TEA a restringir sua ingestão aos alimentos de texturas preferenciais, toleráveis e gerenciáveis. A textura dos alimentos tem sido consistentemente identificada como aspecto relacionado à aceitação de alimentos (HUBBARD KL, et al., 2014; POSTORINO V, et al., 2015). Exemplo disso são os dados de um estudo realizado por Hubbard et al., (2014), cuja amostra foi constituída por 53 crianças com TEA situadas na faixa etária entre 3 e 11 anos, no qual descobriram que a textura e a consistência dos alimentos foram destacados pelos pais dos participantes como razão para a ocorrência de recusa alimentar.

Dessarte, a seletividade alimentar constitui uma problemática relevante e deve ser trabalhada com muito destaque, pois pode propiciar o surgimento de deficiências nutricionais graves, prejudicando o processo de desenvolvimento de crianças com TEA. Logo, torna-se primordial a abordagem multiprofissional, envolvendo médicos especializados e nutricionistas capacitados com vistas a oferecer um tratamento nutricional adequado e aconselhar familiares sobre o comportamento de seus filhos durante as refeições (CERMAK SA, et al., 2010).

Enfatiza-se que o interesse pela temática surgiu ao observar uma carência de informações ofertadas aos pais/cuidadores acerca da importância de uma nutrição adequada. Nesta perspectiva, afirma-se que o presente estudo objetiva analisar a possível presença de comportamentos tendenciosos à seletividade alimentar em crianças com TEA.

\section{MÉTODOS}

Tratou-se de uma pesquisa de campo descritiva, do tipo exploratória, com abordagem quantitativa. 0 cenário desta investigação foi o Município de Caxias, situado na região leste do estado do Maranhão, a 374 quilômetros da capital São Luís, e a 70 quilômetros da capital piauiense, Teresina. Apresenta uma população aproximada de 162.657 mil habitantes, com área da unidade territorial de 5.196,771 km, e densidade demográfica de $30.12 \mathrm{hab} / \mathrm{km}$. A cidade é situada no meio-norte maranhense (IBGE, 2017). Para tanto, utilizou-se como campo de pesquisa a Associação de Pais e Amigos dos Excepcionais (APAE). Ressalta-se que a escolha do local de pesquisa se deu devido ao fato de ser referência de atenção ao público de interesse.

Participaram desta pesquisa crianças com o diagnóstico de TEA atendidas na Associação de Pais e Amigos dos Excepcionais (APAE) na cidade de Caxias- MA. Para determinar o número de participantes foram consideradas informações da gestão do serviço supracitado, as quais demonstraram que existiam cerca de 32 indivíduos com TEA cadastrados e acompanhados pelo serviço supradito, entretanto somente 29 fizeram parte da amostra deste estudo por atenderem aos critérios de inclusão. Portanto, com o intuito de realizar um mapeamento das principais dificuldades na alimentação, todas as mães/responsáveis pelos participantes foram convidadas a compor o estudo.

Os critérios de inclusão para essa pesquisa foram: possuir o diagnostico de TEA, estar recebendo os atendimentos e seguindo os protocolos impostos pelos profissionais responsáveis. Assim, mediante abordagem e realização de convite para participar da presente pesquisa, os participantes foram informados sobre os objetivos do estudo e somente participaram após a aceitação e assinatura do termo de consentimento livre e esclarecido e termo de assentimento. Quanto aos critérios de exclusão, foram desconsiderados aqueles que não eram atendidos na citada instituição ou não estavam frequentando os 
atendimentos. Em relação à coleta de dados, destaca-se que foi aplicado um questionário, com perguntas fechadas para os pais/cuidadores a respeito da alimentação e comportamentos que são característicos de seletividade alimentar, para averiguar a presença deste fator em seus filhos/crianças cuidadas.

Os dados quantitativos, oriundos das questões estabelecida, a partir da aplicação dos questionários aos pais/cuidadores foram tabulados e transformados em tabelas, utilizou-se o programa Microsoft Excel 2013, posteriormente houve análise e interpretação dos dados.

A pesquisa foi submetida à Plataforma Brasil, e, em seguida, direcionada ao Comitê de Ética em Pesquisa (CEP), onde obteve aprovação com número de CAAE 89013318.9.0000.8007. Reitera-se que os pesquisadores se comprometeram com as normas preconizadas pela Resolução do CNS 466/12 e suas complementares, que tratam dos aspectos éticos da pesquisa envolvendo seres humanos.

\section{RESULTADOS E DISCUSSÃO}

\section{Identificação de comportamentos indicativos de seletividade alimentar}

O estudo foi integrado por 29 participantes, nenhum com idade inferior a 3 anos, sendo que a média de idade dos mesmos foi de 9 anos. A partir da análise dos dados, identificou-se que $79,40 \%$ da amostra foi composta por participantes do sexo masculino e $20,60 \%$ são do sexo feminino.

Para a identificação de comportamentos indicativos de seletividade alimentar foi aplicado um questionário com o total de 16 questões acerca da alimentação dos participantes. Esse questionário foi desenvolvido pelos pesquisadores do presente estudo. Reitera-se que o questionário envolve as principais alterações comportamentais presentes nos casos de seletividade alimentar e foi construido embasado em dados da literatura sobre o tema.

Na tabela 1, verifica-se que $85,7 \%$ dos participantes do estudo possuem dificuldades no momento da refeição. Dentro das opções de dificuldades apresentadas pelo questionário, $65,5 \%$ apresentaram dificuldades em consumir novos alimentos e 51,7\% dificuldades com a textura dos alimentos. Quando questionados sobre a variedade alimentar, 16 sujeitos $(52,2 \%)$ responderam que seus filhos não costumam gostar de comer vários tipos de alimentos. Quanto às estrategias utilizadas para melhorar a aceitação de alimentos, um número igual de participantes (16 - 55,1\%) relatou que modificam a apresentação da comida ou negociam o consumo do alimento.

Tabela 1 - Dificuldades apresentada pelos participantes do estudo no momento da refeição. Caxias-MA, 2018.

\begin{tabular}{lcc}
\hline Variáveis & $\%$ & N \\
\hline Dificuldades na hora de se alimentar & & 24 \\
SIM & $85,7 \%$ & 19 \\
Dificuldades em consumir novos alimentos & $65,5 \%$ & 15 \\
Dificuldades com a textura dos alimentos & $51,7 \%$ & 5 \\
NÃO & $14,3 \%$ & 25 \\
Gostam de comer sempre as mesmas coisas? & $89,2 \%$ & 25 \\
SIM & $10,8 \%$ & 4 \\
NÃO & & 16 \\
Consomem vários tipos de alimentos? & $52,2 \%$ & 13 \\
SIM & $44,8 \%$ & \\
NÃO & & 25 \\
São utilizadas estratégia para melhorar a aceitação de alimentos? & $86,2 \%$ & 16 \\
SIM & $55,1 \%$ & 16 \\
"Npresentar o mesmo alimento de varias formas." & $55,1 \%$ \\
NÃO & $13,7 \%$ & 4 \\
\hline
\end{tabular}

Fonte: Dados da pesquisa, 2018. 
Uma característica bastante presente dentro do TEA está relacionada a padrões restritos e repetitivos de comportamento, interesses e atividades, incluindo, então, comportamentos alimentares repetitivos (APA, 2014). A seletividade alimentar (SA) se caracteriza pela junção de comportamentos alimentares, tais como: recusa alimentar, dificuldades em consumir novos alimentos e uma ingestão reduzida de variedades (SILVA $\mathrm{NI}, 2011)$. Portanto, é extremamente importante que tanto os pais quanto os profissionais fiquem atentos quanto a esses comportamentos emitidos no momento da refeição a fim de evitar problemas posteriores (MARTINS RC, 2016).

Parte da recusa, ou mesmo da SA apresentada pela criança com TEA, pode ser justificada por ela não conseguir realizar com eficiência algumas atividades motoras e por possuir distúrbios de processamento sensorial, o que as leva a escolherem ou terem preferência por alimentos através da textura (CORREIA C, 2015).

Um trabalho realizado por Hubbard KL et al. (2014) apresentou relação com o presente estudo, pois identificou que crianças com TEA são mais propensas a apresentarem dificuldades na alimentação, sendo que $77,4 \%$ da amostra recusaram alimentos baseados na textura e consistência que eles apresentaram, este dado corrobora as características identificadas na presente pesquisa. Os autores sugerem que a equipe responsável por atender a crinça deve investigar junto aos pais comportamentos indicativos de seletividade alimentar por meio de anamenese com vistas a direcionar a intervenção e evitar futuros aparecimentos de problemas nutricionais.

O estudo de Bicer AH e Alsaffar AA (2013) identificou que o problema mais comum no momento da refeição era as crianças comerem uma variedade limitada de alimentos pela forte presença de recusa (seletividade alimentar). Além disso, os pais relataram não receber orientações quanto à forma de intervir diante de comportamentos de recusa alimentar.

\section{Problemas de recusa alimentar}

De acordo com dados da tabela 2, 20 participantes do estudo $(68,9 \%)$ costumam recusar alimentos com frequência e dentro das opções ofertadas os alimentos que apresentaram maior nível de recusa foram: vegetais $31,1 \%$, frutas $21,3 \%$ e leites e derivados $14,7 \%$. Ademais, $75,8 \%$ dos participantes costumam escolher os alimentos através da textura, cor e cheiro. Quando mudam a apresentação da comida, 55,1\% dos participantes apresentam comportamentos de resistência, choros, aversões e antipatia (birra).

Tabela 2 - Comportamentos de recusa alimentar dos participantes do estudo. Caxias-MA, 2018.

\begin{tabular}{lll}
\hline Variáveis & $\%$ & $\mathbf{N}$ \\
\hline Recusa alimentos com frequência & & \\
Sim & $68,9 \%$ & 20 \\
Não & $31,1 \%$ & 9 \\
\hline Quais dos alimentos listados, seu filho costuma recusar? & $21,3 \%$ & 13 \\
Frutas & $31,1 \%$ & 19 \\
Vegetais & $14,7 \%$ & 9 \\
Leites e derivados & $75,8 \%$ & 22 \\
\hline Seu filho costuma escolher os alimentos através da textura, cor ou cheiro? & $24,2 \%$ & 7 \\
Sim & & \\
Não & $55,1 \%$ & 16 \\
\hline Seu filho faz birra quando você apresenta um novo alimento, ou quando muda a & $44,9 \%$ & 13 \\
\hline apresentação da comida? &
\end{tabular}

Fonte: Dados da pesquisa, 2018.

O TEA de forma geral é caracterizado por um conjunto de alterações comportamentais, dentro dessas alterações estão presentes dificuldades com as funções executivas: de previsibilidade de saber horários, de se manter na mesa pelo tempo programado para a refeição e isso pode influênciar o interesse da criança 
pelo alimento, uma vez que a mesma pode não conseguir identificar que o momento da refeição é importante (CZERMAINSKI FR, et al., 2013).

Outro fator importante no momento da refeição é que crianças com TEA tendem a ficar sob controle restrito de estímulos, ou seja, respondem sob controle de partes do estímulo ou características irrelevantes deste. Com isso, elas podem apresentar dificuldades de emitir uma resposta a um determinado estímulo, num determinado local, frente a outro estímulo, e isso pode contribuir com a recusa alimentar uma vez que a criança estará respondendo a outros estímulos do ambiente e sem atenção ao que é de interesse no momento da refeição que é o alimento (PORTELA MMFA, 2014).

Além disso, crianças com TEA podem apresentar prejuízo no processamento sensorial e, por conseguinte, possuírem dificuldades de processar algumas informações como: texturas, sabores, cheiros e aspecto visual da comida e, a partir disso, escolherem os alimentos através desses sentidos. É essencial que os pais/cuidadores aprendam a observar os dados relevantes do comportamento do seu filho e valorizar a integração dos sentidos nas ações cotidianas (NASCIMENTO OS, et al., 2015).

Um estudo realizado por Sharp WG et al. (2018), com o objetivo de analisar o risco de inadequação nutricional e variedade alimentar em cianças com TEA, identificou que dois terços da amostra consumiam uma dieta que omitia todos os vegetais e $27 \%$ omitiam todas as frutas. Risco para pelo menos uma inadequação nutricional foi identificada em todas as crianças da amostra do referido trabalho. Dados dessa pesquisa ratificam os achados obtidos no presente estudo, uma vez que a baixa ingestão de vegetais e frutas pode proporcionar um desequilíbrio na ingestão de micronutrientes e levar a inadequação nutricional.

\section{O perfil familiar no momento da refeição}

A partir da análise dos dados contidos na tabela 3, identifica-se que 16 participantes do estudo $(55,1 \%)$ no momento da refeição vivenciam um ambiente silencioso, e que 44,9\% (13) não experimenta a mesma realidade. Quanto aos estimulos sonoros existentes no momento das refeições, 12 participantes (41,3\%) deram destaque para a televisão e 8 participantes $(27,5 \%)$ à presença de conversas de adultos no momento da refeição. Quando questionados sobre o tempo programado para a refeição, $65,6 \%$ da amostra respondeu não ser curto e $34,4 \%$ responderam ser curto.

Tabela 3 - Perfil e ambiente familiar no momento da refeição dos participantes do estudos. Caxias-MA, 2018.

\begin{tabular}{lcc}
\hline Variáveis & $\%$ & $\mathbf{N}$ \\
\hline Na hora da refeição o ambiente é silencioso? & $55,1 \%$ & 16 \\
Sim & $44,9 \%$ & 13 \\
Não & $41,3 \%$ & 12 \\
Televisão & $27,5 \%$ & 8 \\
Conversas de adultos & & \\
\hline Quando seu filho/ pessoa cuidada se suja com a comida ou suja o chão é repreendido? & $34,5 \%$ & 10 \\
Sim & 65,5 & 19 \\
Não & & \\
\hline O tempo programado para as refeições do seu filho é curto? & $34,4 \%$ & 10 \\
Sim & $65,6 \%$ & 19 \\
Não & &
\end{tabular}

Fonte: Dados da pesquisa, 2018.

Segundo Capretz BS et al. (2008), o perfil familiar tem ligação direta com os hábitos alimentares desenvolvidos pela criança, desde o inicio da vida os filhos tendem a copiar as ações dos pais/cuidadores, na alimentação não é diferente. Logo, seguir um modelo de alimentação saudável e considerar o momento da refeição como fonte de prazer e socialização pode contribuir para melhorar tanto as interações sociais de pessoas com TEA como minimizar problemas relacionados a alimentação (CARVALHO JA, et al., 2012). 
Ressalta-se que algumas crianças com TEA podem possuir uma capacidade de atenção reduzida, e as questões relacionadas com controle restrito de estímulos, como discutidas em paragrafo anterior, podem influênciar o comportamento alimentar. Por esse motivo, é importante que os pais/cuidadores estruturem o ambiente onde serão realizadas as refeições, pois a presença de estímulos sonoros e ambientais podem atrapalhar a criança no momento da refeição e desviar sua atenção da comida, contribuindo assim para o desenvolvimento de seletividade alimentar e desinteresse pelo alimento (HORTA A, et al., 2013).

Sobre isso, Valle NMJ e Euclydes PM (2007) identificaram que a formação dos hábitos alimentares na infância sofre a influência dos fatores fisiológicos e ambientais, sendo que dentre os fatores ambientais destacam-se a influência materna (a criança reproduz comportamentos) e da televisão (a televisão é um influenciador de hábitos alimentares, epecialmente alimentos doces e gordurosos).

\section{CONCLUSÃO}

Os resultados deste estudo apontam que os participantes analisados possuem comportamentos tendenciosos à seletividade alimentar. Evidencia ainda risco de problemas nuticionais nas crianças pesquisadas, um fator preocupante, haja vista que a fase da infância tem influência direta a longo prazo. É necessário que essas crianças recebam intervenção adequada no que tange ao acompanhamento nutricional, pois observa-se uma carência de informação ofertada aos pais/cuidadores acerca da importância da alimentação no TEA. Finalmente, sugere-se que estudos posteriores realizem mais investigações acerca da presença de seletividade alimentar e consumo de micronutrientes em indivíduos com TEA.

\section{REFERÊNCIAS}

1. AMERICAN PSYCHIATRIC ASSOCIATION - APA. Manual diagnóstico e estatístico de transtornos mentais: DSM-5. Associação Brasileira de Psiquiatria. Trad. Maria Inês Corrêa Nascimento. Porto Alegre: Artmed, 2014.

2. BAXTER AJ., et al. The epidemiology and global burden of autism spectrum disorders. Revista Psychological Medicine, 2015;45(3):601-613.

3. BICER AH, ALSAFFAR AA. Body mass index, dietary intake and feeding problems of Turkish children with autism spectrum disorder (ASD). Research in Developmental Disabilities, 2013; 34(11):3978-3987.

4. CAPRETZ BS, et al. Variáveis da família e seu impacto sobre o desenvolvimento infantil. Temas em Psicologia, 2008; 16(2).

5. CARVALHO JÁ, et al. Nutrição e autismo: considerações sobre a alimentação do autista. Revista Científica do ITPAC, 2012; 5(1).

6. CERMAK SA, et al. Food selectivity and sensory sensitivity in children with autism spectrum disorders. Journal of the American Dietetic Association, 2010; 110(2):238-246.

7. CORREIA C. Seletividade alimentar e sensibilidade sensorial em crianças com perturbação do espectro do autismo. 2015. Dissertação (Mestrado em Terapia Ocupacional) - Escola Superior de Saúde do Alcoitão, Santa Casa de Misericórdia de Lisboa, Lisboa, 2015.

8. CZERMAINSKI FR, et al. Funções executivas em crianças e adolescentes com transtorno do espectro do autismo: uma revisão. Psicologia Porto Alegre, 2013;44(4):518-525.

9. DOMINGUES G. Relação entre medicamentos e ganho de peso em indivíduos portadores de autismo e outras síndromes relacionadas. Disponível em: <http://www.nutricaoativa.com.br/arquivos/monografia9.pdf >. Acesso em: 03 fev. 2019.

10. ELSABBAGH M, et al. Global Prevalence of Autism and Other Pervasive Developmental Disorders. Revista Autism Research, 2012;5(3):160-179.

11. HORTA A, et al. Marketinge alimentação no espaço escolar: estímulos sensoriais/corporais e a sua apropriação pelas crianças. Revista da Faculdade de Letras da Universidade do Porto, 2013; 85-108.

12. HUBBARD KL, et al. A comparison of food refusal related to characteristics of food in children with autism spectrum disorder and typically developing children. Journal of the Academy of Nutrition and Dietetics, 2014;114(12).

13. IBGE. Instituto Brasileiro de Geografia e Estatística. Estimativas da população residente com data de referência 1ํ de julho de 2017, 2017. 
14. MARTINS RC. O comportamento alimentar e hábitos alimentares da criança em idade pré-escolar: relação com as estratégias parentais. Dissertação (Mestrado em Psicologia) - Faculdade de Psicologia, Universidade de Lisboa, Lisboa, 2016.

15. NASCIMENTO OS, et al.Comportamentos de crianças do Espectro do Autismo com seus pares no contexto de educação musical. Revista Brasileira de Educação Especial, 2015;21(1):93-110.

16. PAULA CS, et al. Epidemiologia e Transtornos Globais do Desenvolvimento, in ARAÚJO JSSC. (Ed.) Transtornos do Espectro do Autismo. 1를. Edão Paulo: Memnon Edições Científicas, 2011, p. 151-158.

17. PORTELA MMFA. Controle restrito de estímulos em autistas: avaliação de um procedimento de Resposta de Observação Diferencial e estímulos com diferenças críticas. Dissertação (Mestrado em Psicologia Experimental) - Pontificia Universidade Católica de São Paulo, São Paulo, 2014.

18. POSTORINO V, et al. Clinical differences in children with autism spectrum disorder with and without food selectivity. Revista Appetite, 2015;92:126-132.

19. SAMPAIO ABM, et al. Seletividade alimentar: uma abordagem nutricional. Revista Brasileira de Psicologia, 2013;62(2):164-170.

20. SHARP WG, et al. Dietary Intake, Nutrient Status, and Growth Parameters in Children with Autism Spectrum Disorder and Severe Food Selectivity: an Electronic Medical Record Review. Journal of the Academy of Nutrition and Dietetics, 2018; 118(10):1943-1950.

21. SILVA NI. Relações entre hábito alimentar e síndrome do espectro autista. Revista Resolução CoPGr 5890 de 2010, 2011.

22. VALLE NMJ, EUCLYDES PM. A formação dos hábitos alimentares na infância: uma revisão de alguns aspectos abordados na literatura nos últimos dez anos. Revista Atenção Primaria à Saúde, 2007;10(1):56-65.

23. WESTWOOD, $\mathrm{H}$, et al. Clinical evaluation of autistic symptoms in women with anorexia nervosa. Molecular Autism, v.8, 2017. 